\title{
Communication \\ Dual-Branch Pre-Distorted Enhanced ADO-OFDM for Full-Duplex Underwater Optical Wireless Communication System
}

\author{
Zixian Wei ${ }^{1}$, Yibin $\mathrm{Li}^{1}$, Zhaoming Wang ${ }^{1}$, Junbin Fang ${ }^{2}$ and Hongyan Fu ${ }^{1, *}$ \\ 1 Tsinghua Shenzhen International Graduate School and Tsinghua-Berkeley Shenzhen Institute (TBSI), \\ Tsinghua University, Shenzhen 518055, China; weizx17@tsinghua.org.cn (Z.W.); \\ liyibin21@mails.tsinghua.edu.cn (Y.L.); wangzm19@mails.tsinghua.edu.cn (Z.W.) \\ 2 Department of Optoelectronic Engineering, Jinan University, Guangzhou 510632, China; \\ tjunbinfang@jnu.edu.cn \\ * Correspondence: hyfu@sz.tsinghua.edu.cn
}

check for

updates

Citation: Wei, Z.; Li, Y.; Wang, Z.; Fang, J.; Fu, H. Dual-Branch Pre-Distorted Enhanced ADO-OFDM for Full-Duplex Underwater Optical Wireless Communication System. Photonics 2021, 8, 368. https:// doi.org/10.3390/photonics 8090368

Received: 17 July 2021

Accepted: 27 August 2021

Published: 1 September 2021

Publisher's Note: MDPI stays neutral with regard to jurisdictional claims in published maps and institutional affiliations.

Copyright: (c) 2021 by the authors. Licensee MDPI, Basel, Switzerland. This article is an open access article distributed under the terms and conditions of the Creative Commons Attribution (CC BY) license (https:// creativecommons.org/licenses/by/ $4.0 /)$.

\begin{abstract}
In this paper, dual-branch pre-distorted enhanced asymmetrically clipped direct current (DC) biased optical orthogonal frequency division multiplexing (PEADO-OFDM) for underwater optical wireless communication (UOWC) is firstly proposed and simulated. The performances of PEADO-OFDM on the underwater optical channel model (UOCM) are analyzed and further compared with the typical ADO-OFDM. Using the Monte Carlo method for the modeling of UOCM, we adopt a double-gamma function to represent three different water qualities including clear, coastal and harbor waters. The full-duplex architecture enables the removal of Hermitian symmetry (HS) from conventional optical OFDM and can increase the spectral efficiency at the cost of hardware complexity. A new PEADO-OFDM transmitter is also proposed to reduce the complexity of the transmitter. The simulation results exhibit that our proposed dual-branch PEADO-OFDM scheme outperforms the typical ADO-OFDM scheme in spectral efficiency, bit error rate (BER) and stability over the underwater channels of three different water qualities.
\end{abstract}

Keywords: pre-distorted enhanced; underwater optical wireless communication (UOWC); ADOOFDM; gamma-gamma function; full-duplex

\section{Introduction}

High-speed underwater communication plays an increasingly important role in oceanographic research, information transfer and marine development [1]. With the application of autonomous underwater vehicles (AUVs), underwater wireless sensor networks (UWSNs) and remotely operated vehicles (ROVs), underwater optical wireless communication (UOWC) with advantages of high bandwidth, cost-effectiveness and low latency becomes a promising wireless communication technology for short/middle reach data exchange compared with acoustic and radio frequency (RF) communications [2,3]. However, seawater, as an enormous and complex physical, chemical and biological system, contains dissolved substances, suspensions and various kinds of active organisms. With the natural characteristics of inhomogeneities of seawater, the transmission light is strongly attenuated because of the absorption and scattering effects that hinder our construction of a precise underwater optical channel model (UOCM) [4,5]. M. Doniec et al. analyzed the spatial distribution of light energy using the volume scattering function [6], and then the Henyey-Greenstein function-based Monte Carlo method was proved to be an effective means [7]. Tang et al. proposed a double-gamma function based on the mentioned Monte Carlo method, which can describe the UOCM relatively precisely and the impulse responses are calculated [8]. The impulse response calculated from the double-gamma function and Monte Carlo simulation has been shown to have a better fitting effect on real underwater models. 
On the other hand, orthogonal frequency division multiplexing (OFDM) is widely used in OWC due to its strong capability to resist inter-symbol interference (ISI). However, the transmitted signals in an OWC system should be non-negative and real-valued due to the intensity modulation and direct detection while the conventional OFDM signals are bipolar complex-valued, which requires the modification of conventional OFDM. Therefore, Hermitian symmetry is commonly utilized before the inverse fast Fourier transform (IFFT) to satisfy the real-valued property. To obtain non-negative signals, various optical OFDM schemes are applied. Direct current biased optical OFDM (DCO-OFDM) adds a DC bias [9], and asymmetrically clipped optical OFDM (ACO-OFDM) utilizes the odd-subcarrier modulation and zero clipping [10]. However, DCO-OFDM and ACO-OFDM suffer from the problems of low power efficiency and low spectral efficiency, respectively. Asymmetrically clipped DC-biased optical OFDM (ADO-OFDM) is the performance tradeoff between DCO-OFDM and ACO-OFDM considering power and spectral efficiency [11-13]. In a conventional ADO-OFDM scheme, the transmitted signals are generated by superimposing the ACO-OFDM signals and DCO-OFDM signals. The zero clipping of the ACO-OFDM branch will introduce clipping noise into the even subcarriers in the transmission of DCOOFDM signals, bringing difficulties to the demodulation of the DCO-OFDM signals. At the receiver, the ACO-OFDM signals must be demodulated first and subtracted from the reconstructed ADO-OFDM signals, and then the DCO-OFDM signals can be demodulated correctly, which results in high-complexity, delay and error propagation issues in demodulation [14]. The pre-distorted enhanced (PE) operation is applied in PEADO-OFDM to decrease the mutual interference and alleviate the issues caused by the clipping noise of the ACO-OFDM branch [15]. PEADO-OFDM eliminates the inter-carrier interference (ICI) between the ACO-OFDM and the DCO-OFDM branches at the transmitter and is applied as the modulation scheme for downlink in a visible light communication (VLC) system. However, the PE operation requires an additional FFT operation at the transmitter, inducing a more complicated transmitter. Compared with Huang's works in ref. [15], we actually proposed a modified scheme in a practical full-duplex UOWC architecture and a low-complexity scheme of a transmitter, apart from transferring the compensation scheme in the ACO branch from a typical indoor model to various underwater channels. The performance comparisons between four optical OFDM schemes from the perspective of spectral efficiency, power efficiency and detection complexity are shown in Table 1.

In this work, we establish a double-gamma UOCM with three kinds of water qualities to observe the performance of the PEADO-OFDM signal in the UOWC system. The fullduplex architecture increases the capacity to exchange information and the stability of alignment in real deployment. By increasing hardware cost, we propose the dual-branch PEADO-OFDM scheme to remove the Hermitian symmetry (HS) operation in the OFDM scheme, which improves the spectral efficiency. A new PEADO-OFDM transmitter with low complexity is further proposed to substitute an absolute operation for the additional FFT operation at the transmitter. For the first time, we apply PEADO-OFDM in the UOCM by further comparing it with a traditional ADO-OFDM scheme in the aspects of bit error rate (BER), stability and spectral efficiency.

Table 1. General performance comparisons between four optical O-OFDM schemes.

\begin{tabular}{cccc}
\hline Optical OFDM Scheme & Spectral Efficiency & Power Efficiency & Detection Complexity \\
\hline DCO-OFDM & high & low & low \\
ACO-OFDM & low & high & low \\
ADO-OFDM & high & medium & high \\
PEADO-OFDM & high & medium & low \\
\hline
\end{tabular}

\section{Channel Model and UOWC System}

\subsection{Underwater Optical Channel Model}

When the light signal propagates in the seawater, the photon suffers from absorption and scattering by the interactions with seawater. The absorption process will reduce the 
energy of the photon while the scattering process will change the transmit direction of the photon. The absorption coefficient $a(\lambda)$ and scattering coefficient $b(\lambda)$ are utilized to evaluate the effects of absorption and scattering, respectively, which vary with water type and wavelength $\lambda$. The total energy loss due to absorption and scattering can be described by the attenuation coefficient $c(\lambda)$, which is given by:

$$
c(\lambda)=a(\lambda)+b(\lambda),
$$

The $a(\lambda), b(\lambda)$ and $c(\lambda)$ for clear, coastal and harbor water used in this paper are listed in Table 2.

Table 2. The values of $a(\lambda), b(\lambda)$ and $c(\lambda)$ for clear, coastal and harbor water.

\begin{tabular}{ccc}
\hline Water Type & $\mathbf{a}\left(\mathbf{m}^{-\mathbf{1}}\right)$ & $\mathbf{b}\left(\mathbf{m}^{-\mathbf{1}}\right)$ \\
\hline Clear Water & 0.114 & 0.037 \\
Coastal Water & 0.179 & 0.219 \\
Harbor Water & 0.295 & 1.875 \\
\hline
\end{tabular}

Referring to the close-form expression of the gamma-gamma function given in [8], the impulse response of UOCM is:

$$
h(t)=C_{1} \Delta t e^{-C_{2} \Delta t}+C_{3} \Delta t e^{-C_{4} \Delta t}
$$

where $\Delta t$ represents the duration of the impulse response.

By using the nonlinear least square criterion:

$$
\left(C_{1}, C_{2}, C_{3}, C_{4}\right)=\arg \min \left(\int\left[h(t)-h_{m c}(t)\right]^{2} d t\right)
$$

where $h(t)$ and $h_{m c}(t)$ are the impulse response of double-gamma functions and Monte Carlo simulation results, respectively, and the operator arg $\min (\cdot)$ is used to return the argument of the minimum.

In the simulation, the Monte Carlo simulations are firstly carried out to obtain the Monte Carlo impulse responses for three kinds of water. Then, Equation (3) can be realized by the curve fitting approach in MATLAB to compute the parameter sets $\left(C_{1}, C_{2}, C_{3}, C_{4}\right)$. By this method, we can build up various impulse responses of clear, coastal and harbor waters. The normalized impulse responses of clear, coastal and harbor waters are shown in Figure 1. We can observe that the time delay spread of the harbor water channel is much larger than that of the other two, which means it will cause more serious ISI.
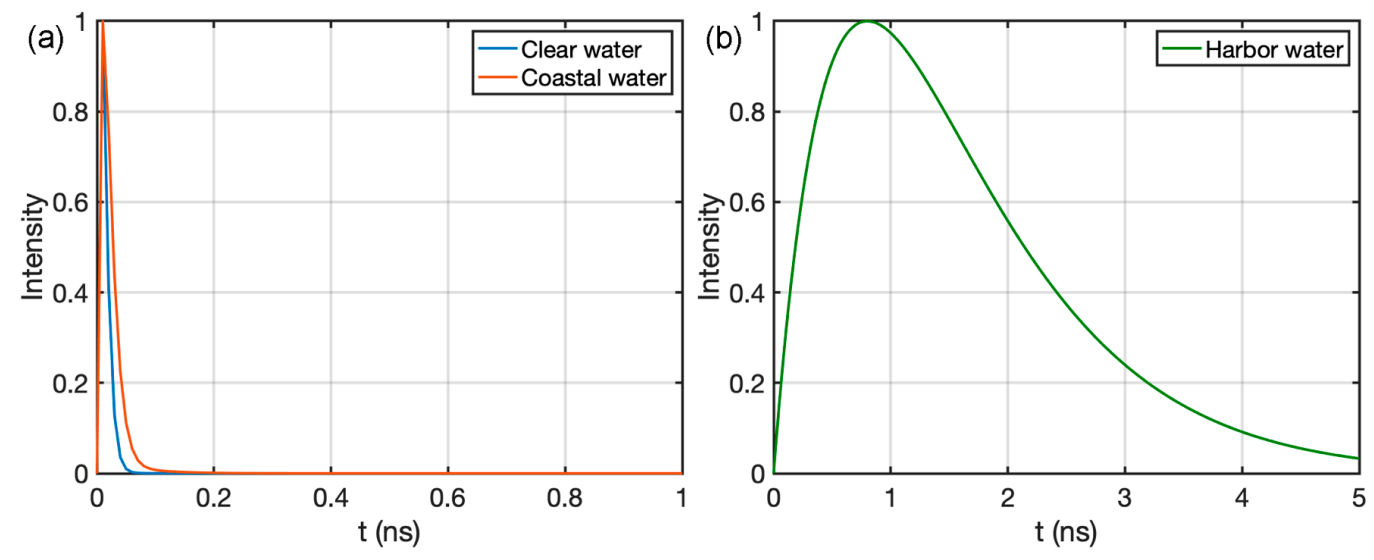

Figure 1. The normalized impulse response in (a) clear, (a) coastal and (b) harbor water using double-gamma functions. 


\subsection{Full-Duplex Architecture}

As shown in Figure 2, the proposed full-duplex UOWC system consists of transceivers, which combine a blue laser diode (LD), a green LD, and two photo-detectors (PDs) within an independent unit. By using this method, blue and green LDs can be used to load the real and imaginary branches of inverse fast Fourier transform (IFFT), respectively. This results in the removal of HS in the optical OFDM scheme. In Figure 2d, the diagonal arrangement of a pair of light sources and a pair of photo-detectors can increase the stability for real applications because the beams can be captured more easily at the receiver side. When both sides adopt this design, a high-speed full-duplex UOWC system will be able to support the requirements of data interaction, simultaneously. For the transmitter and receiver signal processing shown in Figure 2a,b, the details will be introduced in the next section.

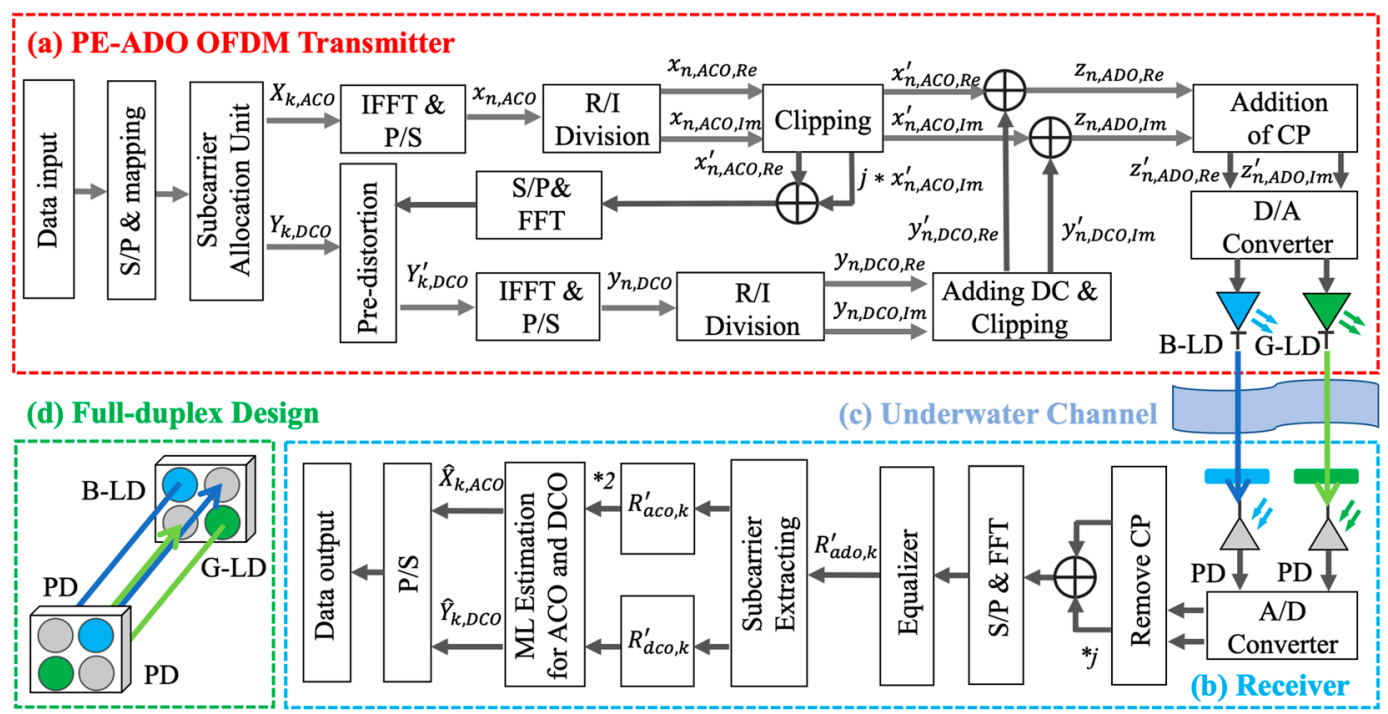

Figure 2. The full-duplex architecture of UOWC system. (a) The dual-branch PEADO-OFDM-based transmitter with blue/green LDs. (b) The dual-branch PEADO-OFDM-based receiver with double PDs. (c) The UOCM. (d) The scheme of full-duplex design.

\section{Proposed PEADO-OFDM Scheme and Full-Duplex Communication}

\subsection{The PEADO-OFDM Scheme}

PEADO-OFDM is a modified ADO-OFDM scheme with subcarrier allocation, in which the ACO-OFDM branch occupies only a part of odd subcarriers and the DCO-OFDM branch occupies the remaining subcarriers except the $0 \_t h$ subcarrier, which improves the flexibility of the system. Therefore, the numbers of subcarriers carrying information assigned to the ACO-OFDM branch and DCO-OFDM branch are not fixed anymore.

In the PEADO-OFDM scheme, the clipping noise from the ACO-OFDM branch will be eliminated at the transmitter. For the ACO-OFDM branch, the time-domain clipped ACO-OFDM signals can be obtained through the traditional ACO-OFDM operations. The noise generated from the clipping operation only falls on the even subcarrier. So, after performing the fast Fourier transform (FFT) operation to the clipped ACO-OFDM signals, the clipping noise can be obtained by extracting the data of the even subcarrier, which will be used as the pre-distorted signals. For the DCO-OFDM branch, before the IFFT operation, the original DCO-OFDM symbols on the even subcarriers should subtract the pre-distorted signals first, which is referred to as the pre-distortion process. Then, the traditional DCOOFDM operations will be performed to obtain the time-domain DCO-OFDM signals.

After superimposing the ACO-OFDM signals and the DCO-OFDM signals in the time domain, the clipping noise that falls on the even subcarriers can be eliminated through pre-distortion, which brings independent demodulation of the ACO-OFDM and DCO- 
OFDM branches, leading to a reduction in the processing latency, complexity and error propagation at the receiver.

\subsection{The Proposed PEADO-OFDM Transmitter with Low Complexity}

The PEADO-OFDM scheme removes the clipping noise at the transmitter side but requires an additional FFT operation. Therefore, we propose another PEADO-OFDM transmitter with low complexity by utilizing the internal characteristics of clipped ACOOFDM signals.

In ACO-OFDM, the input symbols of IFFT can be represented as:

$$
X=\left[0, X_{1}, 0, X_{3}, \ldots, 0, X_{N-1}\right],
$$

where $N$ is the size of IFFT. We denote the output signals of IFFT and the clipped ACOOFDM signals as $x_{n}$ and $x_{n, c}$, respectively. $x_{n, c}$ can be described by $x_{n}$, which is given by:

$$
x_{n, c}=\frac{1}{2}\left(x_{n}+\left|x_{n}\right|\right), 0 \leq n<N,
$$

Applying the FFT operation to both sides of Equation (4), we can obtain:

$$
X_{c}=\frac{1}{2} X+\frac{1}{2} F F T\left(\left|x_{n}\right|\right),
$$

where $X_{c}$ represents the FFT of clipped ACO-OFDM signals $x_{n, c}$. In addition, $X_{c}$ can be divided into even and odd parts, i.e.,:

$$
\begin{gathered}
X_{c}=X_{\text {odd }, c}+X_{\text {even }, c}, \\
X_{\text {odd }, c}= \begin{cases}0, & \text { if } k \text { is even, } \\
X_{c}, & \text { if } k \text { is odd, }\end{cases} \\
X_{\text {even }, c}= \begin{cases}X_{c}, & \text { if } k \text { is even, } \\
0, & \text { if } k \text { is odd, }\end{cases}
\end{gathered}
$$

where $X_{o d d, c}$ and $X_{\text {even,c }}$ represent the odd part and even part of the $X_{c}$, respectively.

It is obvious that $X_{\text {even, },}$ represents the frequency domain form of the clipping noise because the clipping noise only falls on the even subcarrier. For ACO-OFDM signals, it has been proven that after clipping, the symbols on the odd subcarriers are only half of the original symbols in the frequency domain. Thus, $X_{o d d, c}$ can be rewritten as:

$$
X_{o d d, c}=\frac{1}{2} X,
$$

Combining Equations (6), (7) and (10), it is easy to derive that:

$$
X_{\text {even }, c}=\frac{1}{2} F F T\left(\left|x_{n}\right|\right),
$$

Equation (11) reveals that the frequency domain form of the clipping noise $X_{\text {even,c }}$ and the absolute value of the output signals of IFFT are a pair of Fourier transforms. Therefore, we can obtain the clipping noise by employing an absolute operation in the time domain rather than an FFT operation, resulting in lower complexity. The obtained time-domain clipping noise can be used as pre-distortion signals, which will be subtracted by the DCOOFDM branch after the IFFT operation. The diagram of new PEADO-OFDM transmitter with a dual-branch structure is shown in Figure 3. 


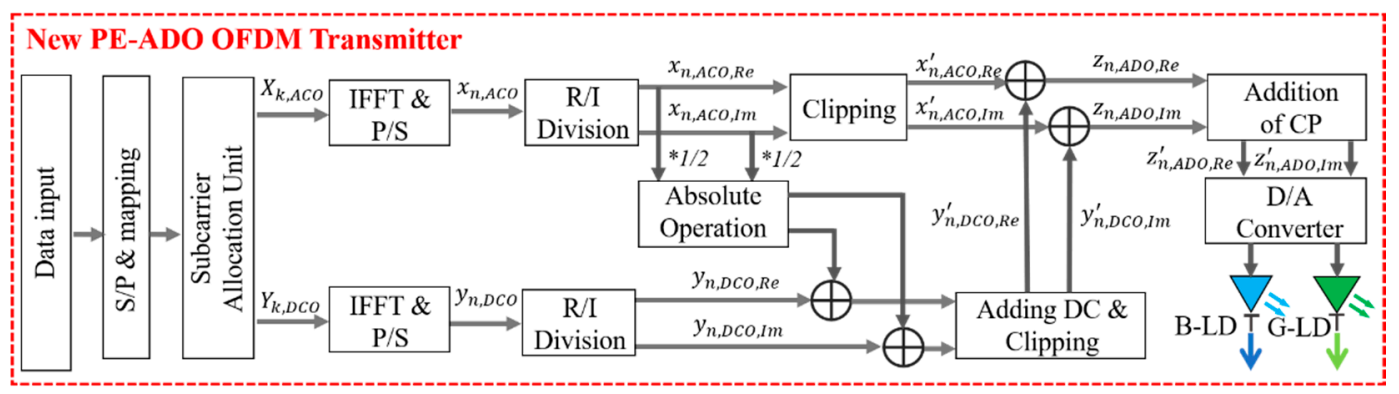

Figure 3. A new PEADO-OFDM transmitter with a dual-branch structure.

\subsection{Dual-Branch PEADO-OFDM Structure}

In O-OFDM, HS is performed before the IFFT to obtain the real-value signals, where the second half of the subcarrier information is the conjugate symmetry of the first half, which greatly reduces the spectral efficiency. Thus, PEADO-OFDM is proposed without HS, so the time-domain signals after the IFFT are complex-value. Then, the real and imaginary branches of the obtained signals will be divided and transmitted independently. For the ACO-OFDM branch, after the real-imaginary division, the negative signals of both the real branch and the imaginary branch will be clipped. For the DCO-OFDM branch, the clipped real and imaginary branches of the ACO-OFDM signals should be combined to the complex-value signals and converted to the pre-distortion signals through the FFT. Then, the operation of pre-distortion, IFFT, S/P and real-imaginary division are performed sequentially. The DC biases are added to both the real and imaginary branches of the pre-distorted DCO-OFDM signals with all the remaining negative signals clipped to zero, which are:

$$
\begin{gathered}
B_{D C, R e}=\mu_{R e} \sqrt{E\left\{y_{n, D C O, R e}^{2}\right\}}, \\
\text { and } B_{D C, I m}=\mu_{I m} \sqrt{E\left\{y_{n, D C O, I m}^{2}\right\}},
\end{gathered}
$$

where $y_{n, D C O, R e}$ and $y_{n, D C O, I m}$ represent the real and imaginary branches of the predistorted DCO-OFDM signals, respectively, and $\mu_{R e}$ and $\mu_{I m}$ represent the bias index of the real and imaginary branches, which can be defined as $\beta_{R e}=10 \log _{10}\left(1+\mu_{R e}^{2}\right)[d B]$ and $\beta_{I m}=10 \log _{10}\left(1+\mu_{I m}^{2}\right)[d B]$, respectively. Hence, by superimposing the real branch from the ACO-OFDM and the DCO-OFDM and the same superimposing operation at the imaginary branch, the real and imaginary branches of the transmitted signals are obtained as:

$$
\begin{gathered}
z_{n, A D C, R e}=x_{n, A C O, R e}^{\prime}+y_{n, D C O, R e}^{\prime} \\
\text { and } z_{n, A D O, I m}=x_{n, A C O, I m}^{\prime}+y_{n, D C O, I m}^{\prime} .
\end{gathered}
$$

Without HS operation, the number of subcarriers conveying information is $N-1$, while in the HS-based O-OFDM scheme, the number of effective subcarriers is $N / 2-1$. Therefore, the spectral efficiency of the proposed transmission scheme is significantly improved.

\section{Results and Discussions}

PEADO-OFDM and ADO-OFDM are simulated and compared in the proposed fullduplex UOWC system with parameters listed in Table 3. In the simulation, a random binary sequence (RBS) is firstly generated and mapped into 4-QAM symbols. Then, the new proposed PEADO-OFDM transmitter structure is applied to generate the PEADOOFDM signals. The signal powers of both PEADO-OFDM and ADO-OFD schemes are normalized to unity. An additive white Gaussian noise model is utilized in the simulation to simulate different $\mathrm{Eb} / \mathrm{N} 0$ between signal and noise. At the receiver, the output binary data 
from the demodulation process will be compared with the transmitted binary sequence to evaluate the system BER.

Table 3. Simulation parameters for dual-branch full-duplex UOWC system.

\begin{tabular}{ccc}
\hline Simulation Parameters & Symbol & Value \\
\hline The number of total subcarriers & $\mathrm{N}$ & 2048 \\
The constellation order & $\mathrm{M}$ & 4 \\
The bias index of the real branch & $\beta_{R e}$ & $10 \mathrm{~dB}$ \\
The bias index of the imaginary branch & $\beta_{I m}$ & $10 \mathrm{~dB}$ \\
Link communication distance & $\mathrm{L}$ & $10 \mathrm{~m}$ \\
The duration of channel impulse response & $\Delta T$ & $5 \mathrm{~ns}$ \\
Bit rate & $\mathrm{bps}$ & $1 \times 10^{9}$ \\
\hline
\end{tabular}

We denote the number of subcarriers assigned to the ACO-OFDM branch as $N_{A C O}$. Here, $N$ is the number of total subcarriers, which is divided into odd subcarriers and even subcarriers for ACO-OFDM and DCO-OFDM, respectively, and the number of subcarriers assigned to the ACO-OFDM branch is fixed to $N / 2$ in traditional ADO-OFDM. In PEADOOFDM, the ACO-OFDM branch only occupies a portion of odd subcarriers so that $N_{A C O}$ can be flexibly adjusted to different sizes, i.e., $N / 2, N / 4$ and $N / 8$. Figure 4 shows the BER comparison of PEADO-OFDM and ADO-OFDM with the proposed scheme in three types of water, where $N_{A C O}$ is set to 1024 , which means that all the odd subcarriers are assigned to the ACO-OFDM branch. Figure $4 \mathrm{a}$,b show that the BER performances in the clear and coastal waters are almost identical. However, in the harbor water, as shown in Figure 4c, the BER performances of both PEADO-OFDM and ADO-OFDM become worse. This result corresponds to the time delay spread shown by the impulse response in Figure 1. Compared with traditional ADO-OFDM in various UOCMs as exhibited in Figure 4, the BER performance of the ACO-OFDM branch in PEADO-OFDM is not changed obviously, while the DCO-OFDM branch in PEADO-OFDM is improved significantly. The PE operation will not affect the demodulation of the ACO-OFDM branch and the DCOOFDM branch can be demodulated independently without depending on the demodulation of the ACO-OFDM branch. Therefore, the overall BER performance of PEADO-OFDM is optimized.

In addition, the BER performances of the DCO-OFDM branch and ACO-OFDM branch with different $N_{A C O}$ are simulated in the clear water, and the simulation results are given in Figure 5a,b, in which $N_{A C O}$ is set to 1024, 512 and 256. It can be seen from the Figure $5 \mathrm{a}$ that with the increase in $N_{A C O}$, the BER performance of the DCO-OFDM branch in PEADO-OFDM will be improved and the required Eb/N0 for a BER of $10^{-3}$ is about $14.80 \mathrm{~dB}, 15.95 \mathrm{~dB}$ and $16.37 \mathrm{~dB}$, achieving about $2.62 \mathrm{~dB}, 1.29 \mathrm{~dB}$ and $0.78 \mathrm{~dB}$ gains compared with that in ADO-OFDM when $N_{A C O}=1024,512$ and 256, correspondingly. The BER performance of the DCO-OFDM branch depends on the channel state and noise when the bias index $\beta$ is large enough. The larger the $N_{A C O}$ is, the more negative ACO-OFDM signals will be clipped, resulting in lower power of the transmitted signals. Therefore, the noise will lower with the same signal-to-noise ratio, leading to a lower BER. 

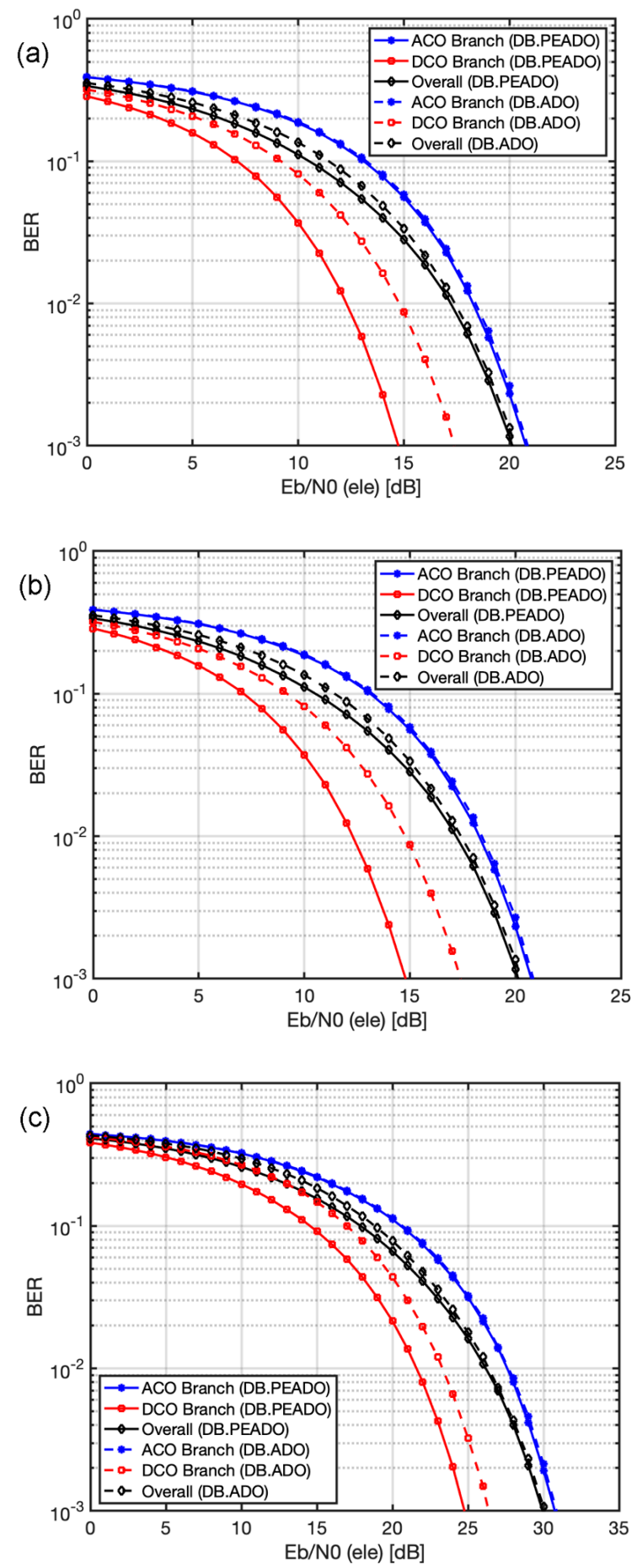

Figure 4. BER performance comparisons of dual-branch ADO-OFDM and PEADO-OFDM in (a) clear, (b) coastal and harbor water channels. (c) the BER performances of both PEADO-OFDM and ADO-OFDM become worse. 

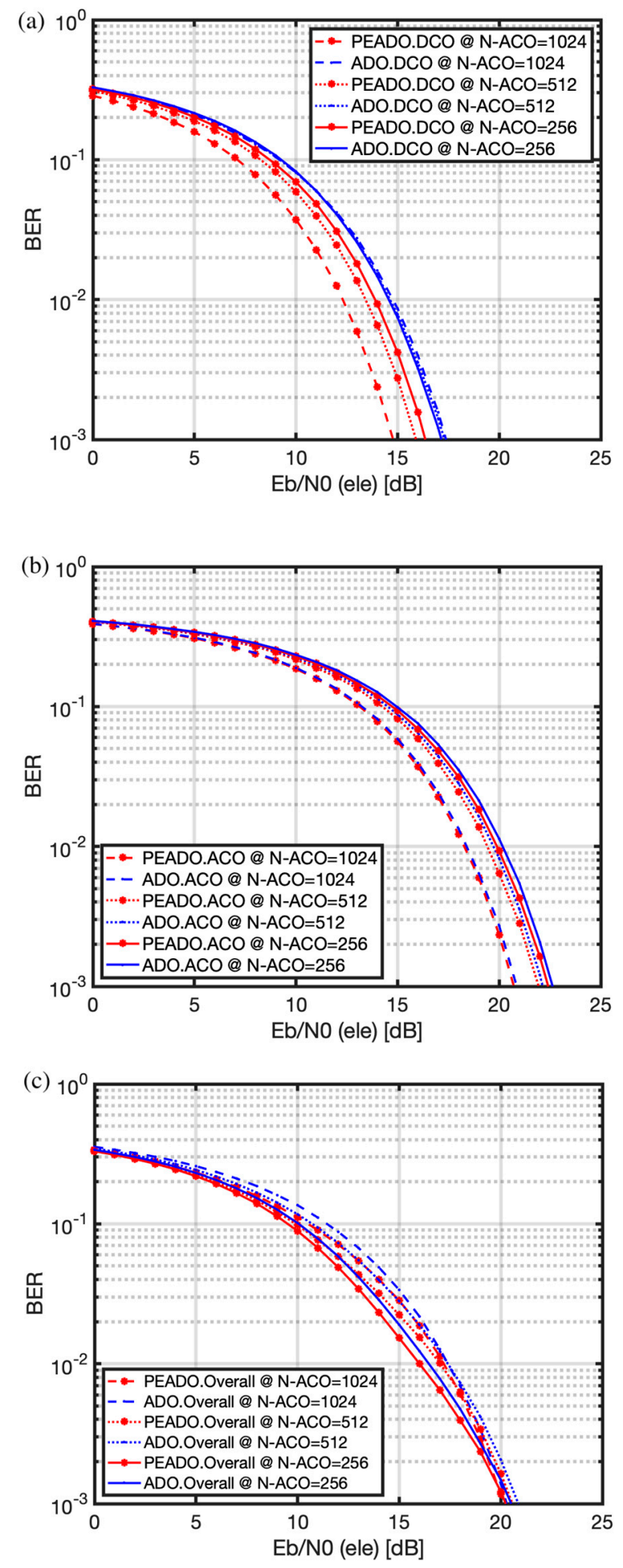

Figure 5. BER performance comparison of (a) the DCO-OFDM branch, (b) the ACO-OFDM branch and (c) the overall dual-branch ADO-OFDM and dual-branch PEADO-OFDM with different $N_{A C O}$ in coastal water channel.

For the ACO-OFDM branch in the PEADO-OFDM and ADO-OFDM schemes, the difference in BER performance becomes obvious when $N_{A C O}$ decreases for the reason that the power of PEADO-OFDM signals is lower than ADO-OFDM signals and the difference is increasing with the decrease in $N_{A C O}$. In Figure $5 c$, the overall BER performances of the PEADO-OFDM and ADO-OFDM schemes with different $N_{A C O}$ in the clear water are performed. For any $N_{A C O}$, the PEADO-OFDM scheme has a better overall BER perfor- 
mance than the conventional ADO-OFDM scheme. To obtain an overall BER of $10^{-3}$, the PEADO-OFDM scheme achieves about $0.16 \mathrm{~dB}, 0.29 \mathrm{~dB}$ and $0.28 \mathrm{~dB}$ gains compared with the enhanced ADO-OFDM scheme when $N_{A C O}=1024,512$ and 256, correspondingly. For the other UOCM channels, the same analysis can obtain similar results.

The proposed modified PEADO-OFDM scheme for the UOWC system not only achieves a lower BER performance on communications, but also allows for easier system scaling. For example, it can be further extended to a multi-channel parallel communication system by using a multi-wavelength transmitter with a blue/green range or spatial division. Such a UOWC system design with the proposed highly efficient scheme proves the communication potential of blue/green LDs, which will play an important role in future high-speed UOWC, integrated systems for underwater drones and underwater information interaction applications.

\section{Conclusions}

In this work, a novel full-duplex architecture UOWC system adopting the dualbranch PEADO-OFDM scheme is investigated. The UOCM with various typical water qualities based on the double-gamma function and Monte Carlo parameter fitting method is introduced for the evaluation. The dual-branch PEADO-OFDM scheme with real and imaginary branches is introduced firstly, and then an improved transmission scheme combining PEADO-OFDM and UOCM is proposed, in which the HS is evitable. The real and imaginary branches of the transmitted time-domain signals can be transmitted via blue and green LDs, respectively. An improved PEADO-OFDM transmitter with low complexity is also proposed. Compared with the traditional ADO-OFDM scheme, our simulation results show that the proposed dual-branch PEADO-OFDM method can improve the spectrum efficiency and the performance of anti-ISI and BER.

Author Contributions: Conceptualization, Z.W. (Zixian Wei); methodology, Z.W. (Zixian Wei); validation, Z.W. (Zixian Wei) and Y.L.; formal analysis, Y.L.; investigation, Y.L.; resources, Z.W. (Zixian Wei); data curation, Z.W. (Zixian Wei); writing-original draft preparation, Z.W. (Zixian Wei), Y.L. and Z.W. (Zhaoming Wang); writing-review and editing, H.F.; visualization, Y.L.; supervision, J.F. and H.F.; project administration, H.F.; funding acquisition, J.F. and H.F. All authors have read and agreed to the published version of the manuscript.

Funding: This research was funded by the Science, Technology and Innovation Commission of Shenzhen Municipality (JCYJ20180507183815699, WDZC20200820160650001); Guangdong Basic and Applied Basic Research Foundation (2021A1515011450); National Natural Science Foundation of China (61771222); and The Fundamental Research Funds for the Central Universities (21620439).

Conflicts of Interest: The authors declare no conflict of interest.

\section{References}

1. Zeng, Z.Q.; Fu, S.; Zhang, H.H.; Dong, Y.H.; Cheng, J.-L. A survey of underwater optical wireless communications. IEEE Commun. Surv. Tutor. 2016, 19, 204-238. [CrossRef]

2. Saeed, N.; Celik, A.; Al-Naffouri, T.Y.; Alouini, M.S. Underwater optical wireless communications, networking, and localization: A survey. Ad Hoc Netw. 2019, 94, 101935. [CrossRef]

3. Cossu, G. Recent achievements on underwater optical wireless communication. Chin. Opt. Lett. 2019, 17, 100009. [CrossRef]

4. Jasman, F.; Green, R.J. Monte Carlo simulation for underwater optical wireless communications. In Proceedings of the 20132 nd International Workshop on Optical Wireless Communications (IWOW), Newcastle Upon Tyne, UK, 21 October 2013 ; pp. 113-117.

5. Sahu, S.K.; Shanmugam, P. A study on the effect of scattering properties of marine particles on underwater optical wireless communication channel characteristics. In Proceedings of the OCEANS 2017-Aberdeen, Aberdeen, UK, 19-22 June 2017; pp. 1-7.

6. Gabriel, C.; Khalighi, M.A.; Bourennane, S.; Léon, P.; Rigaud, V. Monte-Carlo-based channel characterization for underwater optical communication systems. J. Opt. Commun. Netw. 2013, 5, 1-12. [CrossRef]

7. Haltrin, V.I. One-parameter two-term Henyey-Greenstein phase function for light scattering in seawater. Appl. Opt. 2002, 41, 1022-1028. [CrossRef] [PubMed]

8. Tang, S.J.; Dong, Y.H.; Zhang, X.D. Impulse response modeling for underwater wireless optical communication links. IEEE Trans. Commun. 2013, 62, 226-234. [CrossRef]

9. Carruthers, J.B.; Kahn, J.M. Multiple-subcarrier modulation for nondirected wireless infrared communication. IEEE J. Sel. Areas Commun. 1996, 14, 538-546. [CrossRef] 
10. Armstrong, J.; Lowery, A.J. Power efficient optical OFDM. Electron. Lett. 2006, 42, 370-372. [CrossRef]

11. Dissanayake, S.D.; Panta, K.; Armstrong, J. A novel technique to simultaneously transmit ACO-OFDM and DCO-OFDM in IM/DD systems. In Proceedings of the 2011 IEEE GLOBECOM Workshops (GC Wkshps), Houston, TX, USA, 5-9 December 2011; pp. 782-786.

12. Dissanayake, S.D.; Armstrong, J. Comparison of aco-ofdm, dco-ofdm and ado-ofdm in im/dd systems. J. Lightwave Technol. 2013, 31, 1063-1072. [CrossRef]

13. Xuan, H.; Yang, F.; Song, J. Novel heterogeneous attocell network based on the enhanced ADO-OFDM for VLC. IEEE Commun. Lett. 2018, 23, 40-43.

14. Na, Z.; Wang, Y.; Xiong, M.; Liu, X.; Xia, J. Modeling and throughput analysis of an ADO-OFDM based relay-assisted VLC system for 5G networks. IEEE Access 2018, 6, 17586-17594. [CrossRef]

15. Huang, X.; Yang, F.; Pan, C.; Song, J. Pre-Distorted Enhanced ADO-OFDM for Hybrid VLC Networks: A Mutual-Interference-Free Approach. IEEE Photonics J. 2020, 12, 7901512. [CrossRef] 